\title{
SQUARE VARIATION OF BROWNIAN PATHS IN BANACH SPACES
}

\section{MOU-HSIUNG CHANG}

\author{
Department of Mathematics \\ University of Alabama in Huntsville \\ Huntsville, Alabama 35899 \\ U.S.A.
}

ABSTRACT. It is known that if $\{\mathrm{W}(\mathrm{t}), 0 \leq t \leq 1\}$ is a standard Brownian motion in $\mathbb{R}$ then $\lim _{n \rightarrow \infty} \sum_{i=1}^{2 n}\left|w\left(i / 2^{n}\right)-W\left((i-1) / 2^{n}\right)\right|^{2}=1$ almost surely. We generalize this celebrated theorem of Levy to Brownian motion in real separable Banach spaces.

KEY WORDS AND PHRASES. Gaussian measures, abstract Wiener space, Brownian motion in Banach spaces, square variation of Brownian paths.

AMS (MOS) SUBJECT CLASSIFICATIONS (1970). Primary 60J65, 60615, 60617; Secondary $28 A 40$.

\section{INTRODUCTION.}

This note is addressed to those familiar with Levy's theorem [1, p. 510]. It should be readable for those who have an elementary understanding of Banach space and knowledge on Gaussian measure, Bore1-Cantelli lemma, etc.

Let $B$ be a real separable Banach space with norm $\|\cdot\|$ and let $B *$ be the topological dual of B, i.e. the space of bounded linear functionals on $B$. If $\mu$ is a mean zero Gaussian measure on B then it is known [2, p. 35] that B contains a Hilbert space $\mathrm{H}_{\mu}$ with norm $\|\cdot\|_{\mu}$ such that $\|\cdot\|$ is a measurable norm on $H_{\mu}$ in the sense of Gross [2, p. 34], [3, p. 127]. As a consequence, $\|\cdot\|$ is weaker than $\|\cdot\|_{\mu}$. Thus through an injection map it was shown in [2] that the relation $\mathrm{B}^{*} \subset \mathrm{H}^{*} \approx \mathrm{H}_{\mu} \subset \mathrm{B}$ holds. Furthermore, $\mu$ is the extension of a canonical normal distribution on $\mathrm{H}_{\mu}$ to $\mathrm{B}$, and we shall say that $\mu$ is generated by $\mathrm{H}_{\mu}$. 
Let $\left\{\mu_{t}: t \geq 0\right\}$ be the family of Gaussian measures on $B$ given by $\mu_{t}(A)=\delta_{0}(A)$ when $t=0$, and $=\mu\left(A / t^{1 / 2}\right)=\mu\left\{t^{-1 / 2} x: x \in A\right\}$ when $t>0$, where $A$ is any Bore 1 subset of $B$ and $\delta_{0}$ is the unit mass concentrated at the origin of $B$, i.e. $\delta_{0}(\mathrm{~A})=1$ if $0 \in \mathrm{A},=0$ if $0 \notin \mathrm{A}$. Note that $\left\{\mu_{t}: t \geq 0\right\}$ is a semigroup of Gaussian measures under convolution, i.e. $\mu_{t} * \mu_{s}=\mu_{t+s}$, where $\left(\mu_{t} * \mu_{s}\right)(A)=\int_{B} \mu_{t}(A-x) d \mu_{s}(x)$, for any Borel set $A$. Let $\Omega_{B}$ be the space of continuous functions $\omega$ defined on $[0,1]$ into $B$ such that $\omega(0)=0$, and let $F$ be the $\sigma$-field of $\Omega_{B}$ generated by the functions $\omega \rightarrow \omega(t)$. It is clear that $\Omega_{B}$ is a real separable Banach space under the $\|\omega\|_{0}=\sup _{0 \leq t \leq 1}|| \omega(t) \|$, and $F$ coincides with the Borel $\sigma$-field of $\Omega_{B}$. A stochastic process $\{w(t): 0 \leq t \leq 1\}, W(t)(\omega)=w(t)$, on $\Omega_{B}$ is called $\mu$-Brownian motion (restricted to the unit time interval) on $B$ if whenever $0=t_{0}<t_{1}<\ldots<t_{n}=1$, then $\omega\left(t_{j}\right)-\omega\left(t_{j-1}\right) \cdot(j=1,2, \ldots, n)$ are independent and $\omega\left(t_{j}\right)-\omega\left(t_{j-1}\right)$ has distribution $\mu_{t_{j}}-\mu_{t_{j-1}}$ on $B$. Let $P_{W}$ denote the mean zero measure on $\Omega_{B}$ induced by $\{W(t): 0 \leq t \leq 1\} . P_{W}$ is usually called the abstract Wiener measure on $\Omega_{\mathrm{B}}$. Here we shall denote $E_{\mathrm{W}}$ the expectation operator with respect to the measure $\mathrm{P}_{\mathrm{W}}$.

The purpose of this note is to prove an analogue of a celebrated theorem of Lévy [Theorem 5, p. 510] for $\mu$-Brownian motion in a general separable Banach space.

\section{RESULT.}

THEOREM 1. Let $\{W(t): 0 \leq t \leq 1\}$ be $\mu$-Brownian motion in $B$. Then

$$
\lim _{n \rightarrow \infty} \sum_{i=1}^{2^{n}}\left\|w\left(i / 2^{n}\right)-w\left((i-1) / 2^{n}\right)\right\|^{2}=\int_{B}\|x\|^{2} d \mu(x) \text { a.s. }
$$

PROOF. Our proof is elementary and straight-forward, and it goes as follows. Set $s_{n}=\sum_{i=1}^{2^{n}}\left\|w\left(i / 2^{n}\right)-w\left((i-1) / 2^{n}\right)\right\|^{2}, n=0,1,2, \ldots$. Then, from the fact that $\{W(t): 0 \leq t \leq 1\}$ has independent increments and that $r^{-1 / 2} W(r t)$ and $W(t)$ have the same distribution for each $r>0$ and $t>0[3]$, we have 


$$
\begin{aligned}
E_{W}\left(S_{n}\right) & =\sum_{i=1}^{2^{n}} E_{W}\left\|w\left(i / 2^{n}\right)-w\left((i-1) / 2^{n}\right)\right\|^{2} \\
& =\sum_{i=1}^{2^{n}} E_{W}|| w\left(2^{-n}\right) \|^{2} \\
& =\sum_{i=1}^{2^{n}} 2^{-n} E_{W}\|w(1)\|^{2} \\
& =E_{W}|| W(1) \|^{2} \\
& =\left.\int_{B}\|x\|\right|^{2} d \mu(x) .
\end{aligned}
$$

Furthermore,

$$
\begin{aligned}
\operatorname{Var}\left(s_{n}\right) & =\sum_{i=1}^{2^{n}} \operatorname{Var}\left(\left\|w\left(2^{-n}\right)\right\|^{2}\right) \\
& =\sum_{i=1}^{2^{n}} 2^{-2 n} \operatorname{Var}\left(\|w(1)\|^{2}\right) \\
& =2^{-n} \operatorname{Var}\left(\|w(1)\|^{2}\right) .
\end{aligned}
$$

Note that $\operatorname{Var}\left(|| W(1) \|^{2}\right)<\infty$ by a theorem of Fernique [4]. Therefore, by Chebyshev's inequality, we have $P_{W}\left\{\left|S_{N}-E_{W}\left(S_{n}\right)\right|>1 / n\right\} \leq n^{2} \operatorname{Var}\left(S_{n}\right)=n^{2} 2^{-n} \operatorname{Var}\left(|| W(1)||^{2}\right)$. $\sum n^{2} 2^{-n}<\infty$, and the theorem follows from the Borel-Cantelli lemma [5, p. 76]. $\mathrm{n}=1$

REMARK. When $B=\mathbb{R}$ Theorem 1 reduces to that of Levy for the standard Brownian motion, since

$$
\left.\int_{B}|| x\right|^{2} d \mu(x)=\frac{1}{\sqrt{2 \pi}} \int_{-\infty}^{\infty} x^{2} \exp \left\{-x^{2} / 2\right\} d x=1
$$

\section{REFERENCES}

1. LÉVY, P. Le Mouvement brownien plan, Amer. J. Math. 62 (1940), 487-550.

2. GROSS, L. Abstract Wiener space, Proceeding of 5th Berkeley Symposium on Math. Stat. and Prob. 2 (1965), 31-42.

3. GROSS, L. Potential theory on Hilbert space, J. Functional Analysis 1 (1967), 123-181.

4. FERNIQUE, X. Integrabilité des Vecteurs Gaussiens, C.R. Acad. Sci., Paris, Ser. A 270 (1970), 1698-1699.

5. CHUNG, K.L. A Course in Probability Theory, 2nd edition, Academic Press, New York, 1974. 


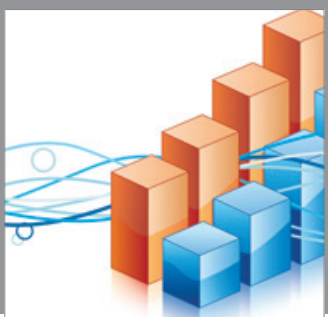

Advances in

Operations Research

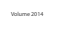

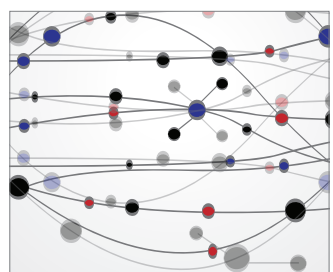

\section{The Scientific} World Journal
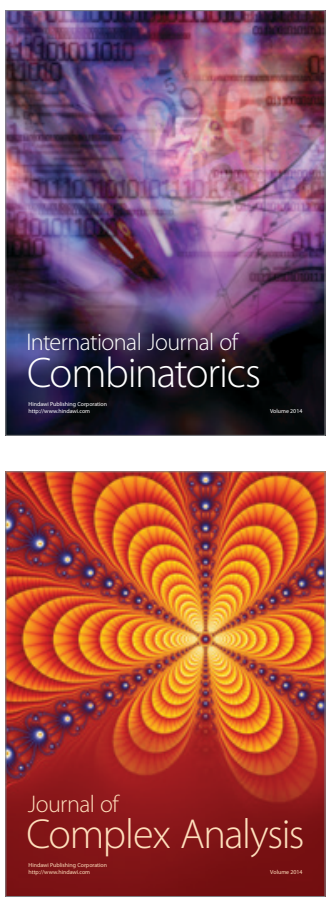

International Journal of

Mathematics and

Mathematical

Sciences
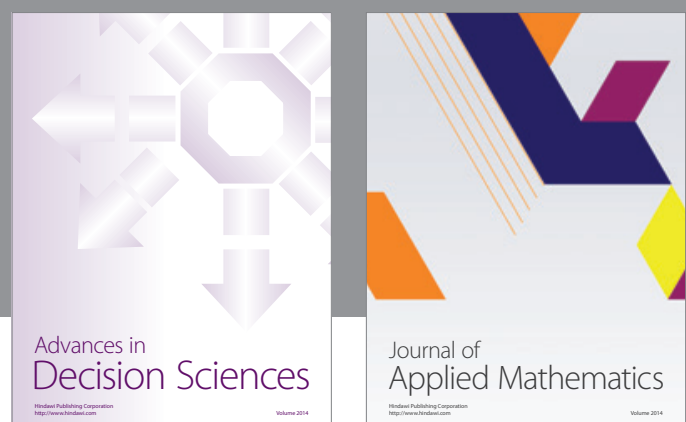

Journal of

Applied Mathematics
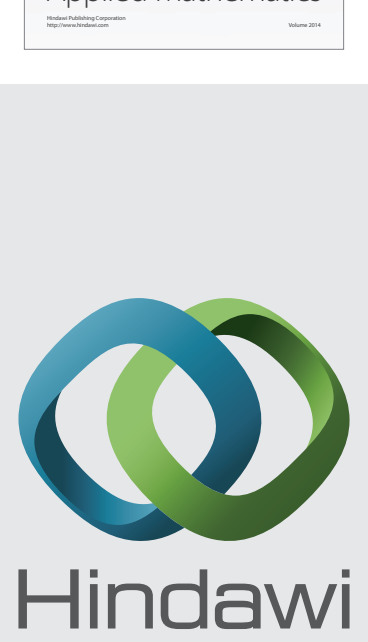

Submit your manuscripts at http://www.hindawi.com
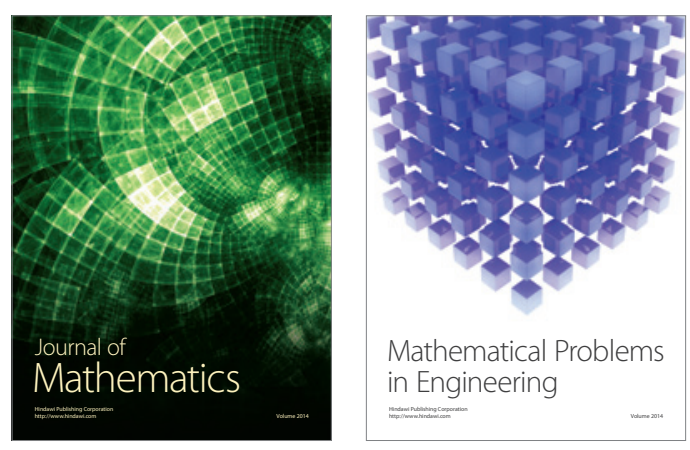

Mathematical Problems in Engineering
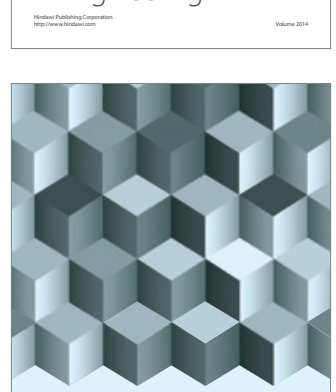

Journal of

Function Spaces
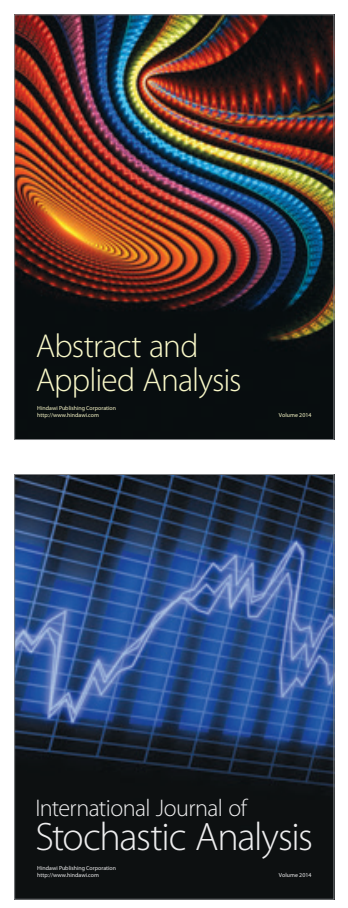

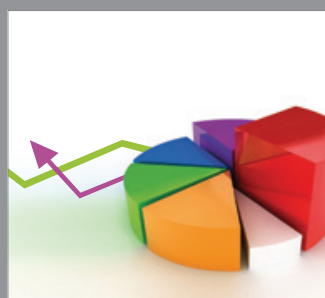

ournal of

Probability and Statistics

Promensencen
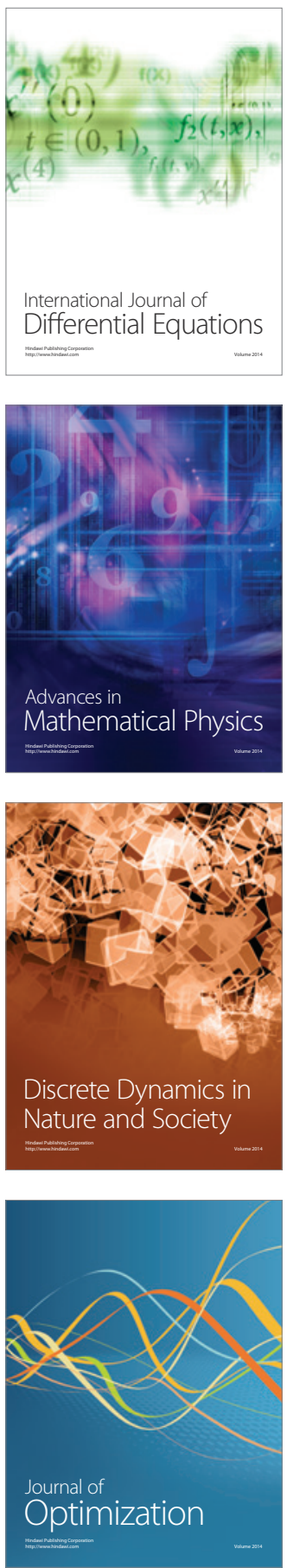